\title{
Persistent Spin Current in a Mesoscopic Hybrid Ring with Spin-Orbit Coupling
}

\author{
Qing-feng Sun, ${ }^{1}$ X. C. Xie, ${ }^{2,1}$ and Jian Wang ${ }^{3, *}$ \\ ${ }^{1}$ Beijing National Lab for Condensed Matter Physics and Institute of Physics, Chinese Academy of Sciences, Beijing 100080, China \\ ${ }^{2}$ Department of Physics, Oklahoma State University, Stillwater, Oklahoma 74078, USA \\ ${ }^{3}$ Department of Physics and the Center of Theoretical and Computational Physics, The University of Hong Kong, Hong Kong, China
}

(Received 12 May 2006; published 7 May 2007)

\begin{abstract}
We investigate the equilibrium property of a mesoscopic ring with a spin-orbit interaction. It is well known that, for a normal mesoscopic ring threaded by a magnetic flux, the electron acquires a Berry phase that induces the persistent (charge) current. Similarly, the spin of an electron acquires a spin Berry phase traversing a ring with a spin-orbit interaction. It is this spin Berry phase that induces a persistent spin current. To demonstrate its existence, we calculate the persistent spin current without an accompanying charge current in the normal region in a hybrid mesoscopic ring. We point out that this persistent spin current describes the real spin motion and can be observed experimentally.
\end{abstract}

DOI: 10.1103/PhysRevLett.98.196801

PACS numbers: 73.23.Ra, 71.70.Ej, 72.25.-b

Recently, the physics of semiconductors with a spinorbit interaction (SOI) has attracted a lot of attention, as it plays an important role for the emerging field of semiconductor spintronics [1]. SOI couples the spin degree of freedom of electrons to their orbital motions, thereby giving rise to a useful way to manipulate and control the electron spin by an external electric field or a gate voltage. Interesting effects resulting from SOI have been predicted. For example, using the effect of spin precessions due to the Rashba SOI, Datta and Das proposed a spin-transistor more than ten years ago [2]. Very recently, a very interesting effect, the intrinsic spin Hall effect, was theoretically predicted by Murakami et al. [3] and Sinova et al. [4], where a substantial amount of dissipationless spin current can be generated from the interplay of the electric field and the SOI. Since then, the spin Hall effect has generated tremendous interest with a great deal of work focusing on the field of spintronics.

In this Letter, we explore another interesting effect that a persistent spin current without the accompanying charge current exists in a coherent mesoscopic semiconductor ring with simplectic symmetry, i.e., with SOI but maintaining the time-reversal symmetry. More than two decades ago, the persistent (charge) current in a mesoscopic ring threaded by a magnetic flux was predicted theoretically [5] and later observed experimentally in the early 1990s [6]. It is now well known that the persistent charge current is a pure quantum effect and can sustain without dissipation in the equilibrium case. There have also been many investigations on the persistent spin current [7-10]. For example, in a mesoscopic ring with a crown-shape inhomogeneous magnetic field [7] or threaded by a magnetic flux [8], the persistent spin current has been predicted and is related to the Berry phase. Recently, the persistent spin current carried by bosonic excitations has also been predicted in a Heisenberg ring with a magnetic field or in a ferromagnetic material [9]. The reason that the persistent spin current exists may be explained as follows. Because of the magnetic field or the magnetic flux, there are persistent flows of both spin up and down electrons. In the absence of SOI, this gives rise to the well-known persistent charge current. In the presence of a SOI or a magnetic field, the persistent charge current is spin polarized, resulting a nonzero persistent spin current. Hence, the origin of this persistent spin current is the same as that of the persistent charge current, so a persistent spin current always accompanies a persistent charge current.

Up to now, the issue of whether the persistent spin current without an accompanying charge current (a pure persistent spin current) can be induced solely by SOI at zero magnetic flux or a magnetic field has not been addressed [11]. In this Letter, we show that a nonmagnetic semiconducting ring with a SOI can sustain a pure persistent spin current in the absence of the external magnetic field or a magnetic flux. Since the magnetic flux or magnetic field acts like a "driving force" for the persistent charge current, one naturally looks for the analogous driving force in the spin case. To discuss this question, let us consider two devices. The first device consists of a mesoscopic ring (without a SOI) where a magnetic atom with a magnetic dipole moment is placed at the center of the ring [see Fig. 1(a)]. In the second device, the magnetic atom is replaced by a charged atom, e.g., an ion [see Fig. 1(b)]. The magnetic atom produces a vector potential $\mathbf{A}$ on the perimeter of the ring which drives the persistent charge current. By analogy, a charged atom which produces a scalar potential $\phi$ on the perimeter of the same ring should drive a persistent spin current. Since the presence of this ionic center generates a SOI in the relativistic limit, we expect that a SOI which plays the role of the spin driving force will induce a pure persistent spin current.

The existence of the pure persistent spin current can be examined from another point of view using the Berry phase. It is well known that an electron circulating a ring with a nonuniform magnetic field or magnetic flux acquires a geometric phase (Berry phase) [12]. It has been discov- 
(a)
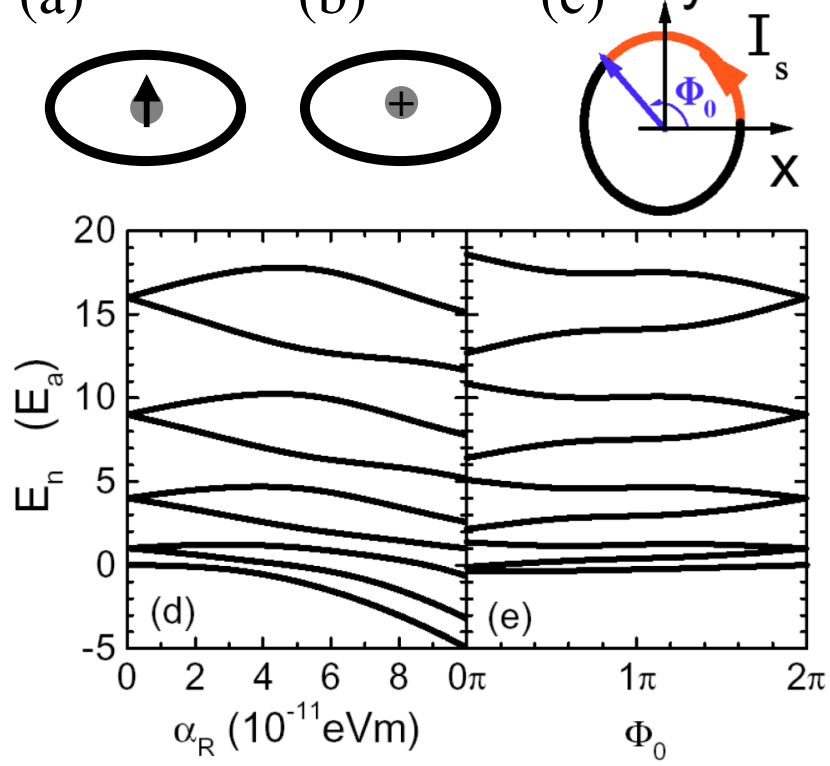

FIG. 1 (color online). (a),(b) are the schematic diagrams for a mesoscopic ring with a magnetic atom or an ion at its center. (c) Schematic diagram for a hybrid mesoscopic ring having Rashba SOI in part of the ring and the other part being normal. (d),(e) show the eigenenergies $E_{n}$ vs $\alpha_{R}$ for $\Phi_{0}=\pi$ and vs $\Phi_{0}$ for $\alpha_{R}=3 \times 10^{-11} \mathrm{eV} \mathrm{m}$, respectively. The ring's radius $a=$ $50 \mathrm{~nm}$.

ered by Loss et al. that it is this Berry phase $\chi$ that induces the well-known persistent charge current [7]. Assuming that the electron wavelength is much smaller than the perimeter of the ring and the electron motion is quasiclassical, let us examine an electron with spin $\sigma$ traversing slowly along the ring with only a SOI [13]. Because of the SOI, the spin of this electron precesses and acquires a geometric phase after the electron returns to its starting point [13]. This is the so-called spin Berry phase [13]. The spin Berry phase due to Rashba SOI for an electron with spin $\sigma$ moving in the clockwise direction is found to be [13] $\chi_{\sigma}=\sigma \pi$, where $\sigma= \pm$ for $\sigma=\uparrow, \downarrow$. From the physical picture due to Loss et al. [7], the spin Berry phase $\chi_{+}$ for the spin up electron induces a clockwise persistent spin polarized current $I_{1}$. Similarly, the Berry phase $\chi_{\text {- induces }}$ a counterclockwise persistent spin polarized current with the polarization exactly opposite to that of $I_{1}$ since our system has time-reversal symmetry. As a result, the spin Berry phase due to a SOI will induce a pure persistent spin current.

Now we present an example to show that indeed a pure persistent spin current can exist for a semiconducting ring with a SOI. In the presence of a SOI, the spin of an electron experiences a torque, and, hence, $\sigma_{i}(i=x, y, z)$ is not a good quantum number anymore. Because of this, the spin current is not conserved using the conventional definition. At present, there are controversies on whether one should define a conserved spin current or whether there exists a conserved spin current [14-17]. In other words, so far there is no consensus on the definition for the spin current in the presence of a SOI. Since the purpose of our Letter is not addressing the controversial issue of the definition of spin current but focusing on the new phenomenon-persistent spin current-we use the following approach. We first discuss the persistent spin current for a one-dimensional mesoscopic semiconducting ring that consists of a Rashba $\mathrm{SO}$ coupling region and a normal region without a SOI as shown in Fig. 1(c). Since there is no spin-flip in this normal region, the spin current can be calculated using the conventional definition without controversy. Then we discuss how to make the spin current conserved and its consequence on the persistent spin current.

The Hamiltonian of our system is given by $[8,18]$

$$
\begin{aligned}
H= & -E_{a} \frac{\partial^{2}}{\partial \varphi^{2}}-\frac{i \sigma_{r}}{2 a}\left[\alpha_{R}(\varphi) \frac{\partial}{\partial \varphi}+\frac{\partial}{\partial \varphi} \alpha_{R}(\varphi)\right] \\
& -i \frac{\alpha_{R}(\varphi)}{2 a} \sigma_{\varphi},
\end{aligned}
$$

where $E_{a}=\hbar^{2} / 2 m a^{2}, a$ is the radius of the ring, $m$ is the effective mass of the electron, $\sigma_{r}=\sigma_{x} \cos \varphi+\sigma_{y} \sin \varphi$, and $\sigma_{\varphi}=-\sigma_{x} \sin \varphi+\sigma_{y} \cos \varphi \cdot \alpha_{R}(\varphi)$ is the strength of the Rashba SOI, $\alpha_{R}(\varphi)=0$, while $0<\varphi<\Phi_{0}$, i.e., in the normal region, and $\alpha_{R}(\varphi)$ is a constant $\alpha_{R}$ in the SO coupling region with $\Phi_{0}<\varphi<2 \pi$.

The eigenstates of Hamiltonian (1) can be solved numerically in the following way. First, in the Rashba SO coupling region $\left(\alpha_{R} \neq 0\right)$, the equation $H \Psi(\varphi)=E \Psi(\varphi)$ has four independent solutions $\Psi_{i}^{\mathrm{SO}}(\varphi)(i=1,2,3,4)[8]$ :

$$
\Psi_{1 / 2}^{\mathrm{SO}}(\varphi)=\left(\begin{array}{c}
\cos (\theta / 2) e^{i k_{1 / 2} \varphi} \\
-\sin (\theta / 2) e^{i\left(k_{1 / 2}+1\right) \varphi}
\end{array}\right)
$$

and $\Psi_{3 / 4}^{\mathrm{SO}}=\hat{T} \Psi_{1 / 2}^{\mathrm{SO}}$, with $\hat{T}$ being the time-reversal operator. In Eq. (2), the wave vectors $k_{1 / 2}=-1 / 2+$ $1 /(2 \cos \theta) \pm(1 / 2) \sqrt{\left(1 / \cos ^{2} \theta\right)-1+4 E / E_{a}}$, and the angle $\theta$ is given by $\tan (\theta)=\alpha_{R} /\left(a E_{a}\right)$. Similarly, in the normal region $\left(0<\varphi<\Phi_{0}\right)$, the Schödinger equation has four independent solutions: $\quad \Psi_{1}^{N}(\varphi)=(1,0)^{\dagger} e^{i k \varphi}$, $\Psi_{2}^{N}(\varphi)=(1,0)^{\dagger} e^{-i k \varphi}$, and $\Psi_{3 / 4}^{N}=\hat{T} \Psi_{1 / 2}^{N}$. Second, the eigenwave function $\Psi(\varphi)$ with the eigenenergy $E$ can be represented as

$$
\Psi(\varphi)= \begin{cases}\sum_{i} a_{i} \Psi_{i}^{N}(\varphi) & \text { while } 0<\varphi<\Phi_{0}, \\ \sum_{i} b_{i} \Psi_{i}^{S O}(\varphi) & \text { while } \Phi_{0}<\varphi<2 \pi\end{cases}
$$

where $a_{i}$ and $b_{i}(i=1,2,3,4)$ are constants to be determined by the boundary conditions at the interfaces $\varphi=0$ and $\Phi_{0}$. Here the boundary conditions are the continuity of the wave function $\left.\Psi(\varphi)\right|_{\varphi=0^{+} / \Phi_{0}^{+}}=\left.\Psi(\varphi)\right|_{\varphi=2 \pi^{-} / \Phi_{0}^{-}}$and the continuity of its flux $\left.\hat{v}_{\varphi} \Psi\right|_{\varphi=0^{+} / \Phi_{0}^{+}}=\left.\hat{v}_{\varphi} \Psi\right|_{\varphi=2 \pi^{-} / \Phi_{0}^{-}}$, where $\hat{v}_{\varphi}=\left(2 a E_{a} / i \hbar\right)\left[\partial / \partial \varphi+(i / 2) \sigma_{r} \tan (\theta)\right]$ is the ve- 
locity operator. Figure 1(d) shows $E_{n}$ versus the Rashba SO strength $\alpha_{R}$. For the normal ring $\left(\alpha_{R}=0\right)$, the eigenvalues are $n^{2} E_{a}$ with fourfold degeneracy, and the corresponding eigenstates are $(1,0)^{\dagger} e^{ \pm i n \varphi}$ and $(0,1)^{\dagger} e^{ \pm i n \varphi}[19]$. As the SOI is turned on, the degenerate energy levels split while maintaining twofold Kramers degeneracy. The higher the energy level, the larger this energy split. Typically, the splits are on the order of $E_{a}$ at $\alpha_{R}=10^{-11} \mathrm{eV} \mathrm{m}$, with $E_{a} \approx 0.42 \mathrm{meV}$ for the ring's radius $a=50 \mathrm{~nm}$ and the effective mass $m=0.036 m_{e}$. The eigenvalues $E_{n}$ versus the normal region's angle $\Phi_{0}$ are also shown [see Fig. 1(e)]. For $\Phi_{0}=2 \pi$, the whole ring is normal and $E_{n}$ are fourfold degenerate [19]. When $\Phi_{0}$ is away from $2 \pi$, the degeneracy is split into two, and the splits are larger with the smaller $\Phi_{0}$. When $\Phi_{0}=0$, the whole ring has the Rashba SOI, and the split reaches the maximum.

Since $E_{n}$ is twofold degenerated, we obtain two eigenstates for each $E_{n}$, which are labeled $\Psi_{n}(\varphi)$ and $\hat{T} \Psi_{n}(\varphi)$. With the wave functions, the spin current contributed from the level $n$ can be calculated straightforwardly using the conventional definition $I_{S i}^{n}(\varphi)=\operatorname{Re} \Psi_{n}^{\dagger} \hat{\boldsymbol{v}}_{\varphi} \hat{\sigma}_{i} \Psi_{n} \quad(i=$ $x, y, z)$. Since there is a controversy about the definition of spin current in the SO region, we will calculate the spin current only in the normal region. We note that the spin current is conserved in the normal region independent of the angle coordinate $\varphi$. Figure 2 shows the spin current $I_{S i}^{n}$ versus the Rashba SO strength $\alpha_{R}$ for $\Phi_{0}=\pi$. Our results show that $I_{S x}^{n}$ is exactly zero for all levels $n$, and $I_{S y}^{n}$ and $I_{S z}^{n}$ exhibit the oscillatory pattern with $\alpha_{R}$. A $\pi / 2$-phase shift between $I_{S y}^{n}$ and $I_{S z}^{n}$ is observed with $\sqrt{\left(I_{S y}^{n}\right)^{2}+\left(I_{S z}^{n}\right)^{2}}$ approximately constant. For two adjacent levels $2 n-1$ and $2 n$, their spin current have opposite signs, and $I_{S i}^{2 n-1}+$ $I_{S i}^{2 n}=0$ if $\alpha_{R}=0$. We note that the spin current $I_{S i}^{n}$ is quite large. For example, the value $E_{a}$ is equivalent to the spin current of a moving electron in the ring with its speed $4 \times 10^{5} \mathrm{~m} / \mathrm{s}$.

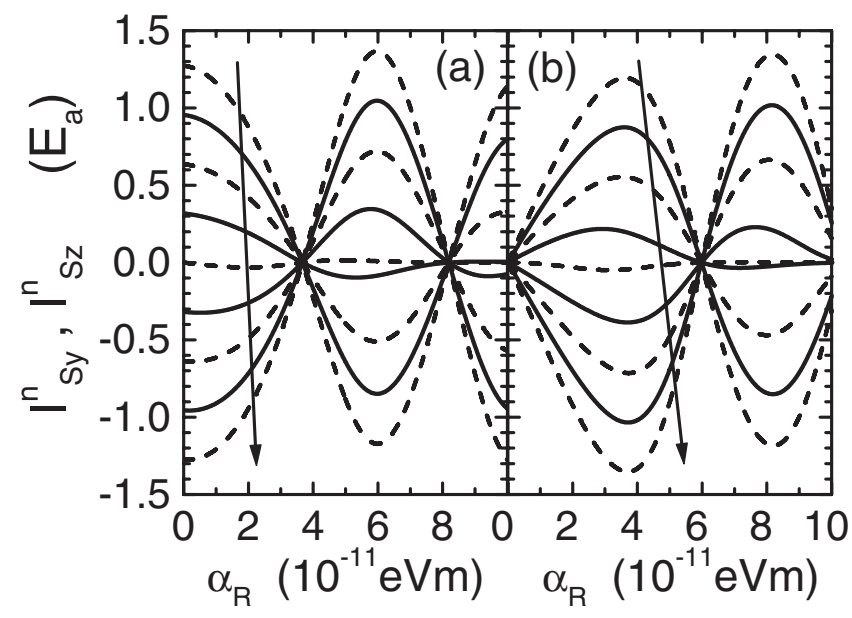

FIG. 2. (a) $I_{S y}^{n}$ and (b) $I_{S z}^{n}$ vs $\alpha_{R}$ for $\Phi_{0}=\pi$ and $a=50 \mathrm{~nm}$. Along the arrow direction, $n=7,5,3,1,0,2,4,6$, and 8 .
Now we calculate the equilibrium total spin current $I_{S i}$ contributed from all occupied energy levels: $I_{S i}=$ $2 \sum_{n} I_{S i}^{n} f\left(E_{n}\right)$, where $f(E)$ is the Fermi distribution and the factor 2 is due to the Kramers degeneracy. The persistent charge current and the equilibrium spin accumulation are found to be zero because the system has the timereversal symmetry. Figures 3(a) and 3(b) show the total spin currents $I_{S i}$ versus the Rashba SO strength $\alpha_{R}$ for different Fermi energy $E_{F}$. One of the main results is that the spin current indeed is nonzero when $\alpha_{R} \neq 0$. The persistent spin currents $I_{S i}$ in Fig. 3 have the following features. At $\alpha_{R}=0$, the whole ring is normal, so $I_{S i}$ is exactly zero. With $\alpha_{R}$ increasing, $I_{S i}$ first increases, and it then oscillates for the large $\alpha_{R}$. At certain $\alpha_{R}$, there is a jump in the curve of $I_{S i}$ versus $\alpha_{R}$, because for this $\alpha_{R}$ the Fermi energy $E_{F}$ is in line with a level $E_{n}$, leading to a change of its occupation. At zero temperature, the jump is abrupt as shown in Figs. 3(a) and 3(b). But at finite temperature, this jump will be slightly smooth. In fact, these results are similar with the persistent (charge) current in the mesoscopic ring [5].

The spin current $I_{S i}$ versus the angle of normal region $\Phi_{0}$ at a fixed $\alpha_{R}=3 \times 10^{-11} \mathrm{eV} \mathrm{m}$ is shown in Fig. 3(c). While $\Phi_{0}=2 \pi$, the whole ring is normal, and $I_{S x / y / z}=0$. When $\Phi_{0}$ is away from $2 \pi$, the spin current $I_{S x / y / z}$ emerges. There perhaps exists a jump in the curve $I_{S i}-\Phi_{0}$ which behaves similarly to the jump in the curve $I_{S i}{ }^{-} \alpha_{R}$. In particular, while $\Phi_{0}$ tends to zero, i.e., the normal region tends to vanish, the spin currents $I_{S x}$ and $I_{S z}$ still exist. This means that the normal region is not necessary for generating $I_{S i}$.

Now we examine the effect of a sharp interface. Let us consider a hybrid ring device with a SOI varying continuously along the ring. The Hamiltonian is the same as

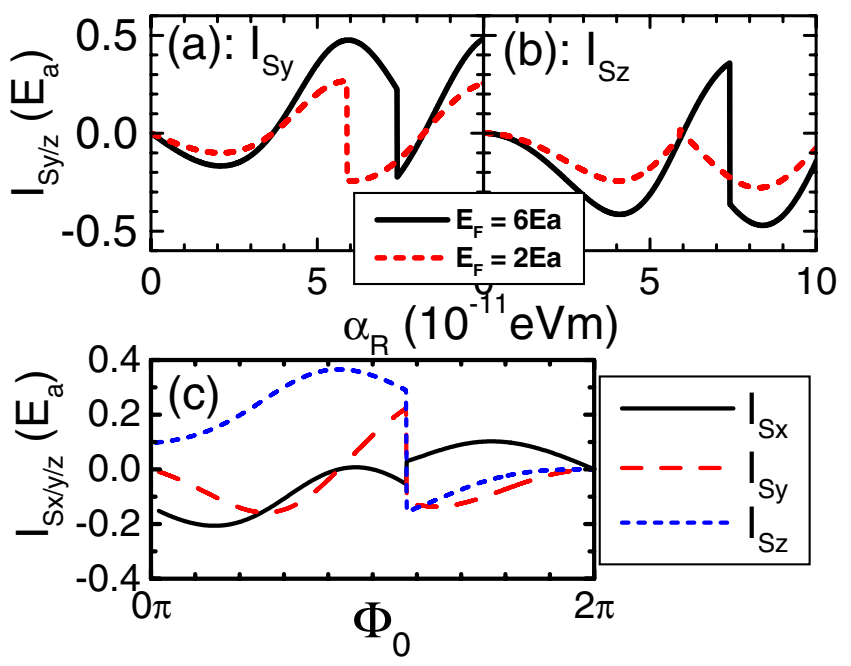

FIG. 3 (color online). (a),(b) show $I_{S y}$ and $I_{S z}$ vs $\alpha_{R}$ for $\Phi_{0}=$ $\pi$. (c) shows $I_{S x / y / z}$ vs the normal region's angle $\Phi_{0}$ for $\alpha_{R}=$ $3 \times 10^{-11} \mathrm{eVm}$ and $E_{f}=3 E_{a}$. The ring's radius $a=50 \mathrm{~nm}$, and the temperature $T=0$. 
Eq. (1), with $\alpha_{R}(\varphi)=0$ for $0<\varphi<\pi$ and $\alpha_{R}(\varphi)=$ $\alpha_{R} \sin ^{2}(\varphi)$ for $\pi<\varphi<2 \pi$. In this case, both $\alpha_{R}(\varphi)$ and $d \alpha_{R}(\varphi) / d \varphi$ are continuous at the interfaces $\varphi=0$ and $\pi$. Our numerical results show that the persistent spin current in the normal region is nonzero, which gives additional support to our conclusion.

Now we address the issue of conservation of spin current. The reason that spin current is not conserved is because there is a torque due to a SO acting on the spin. This can be seen from the continuity equation [14] $-\nabla \cdot \vec{j}_{S i}+$ $j_{\omega i}=0$, where $\vec{j}_{S i}=\operatorname{Re}\left\{\Psi^{\dagger} \hat{\vec{v}} \hat{\sigma}_{i} \Psi\right\}$ is the conventional spin current with spin polarization along direction $i=$ $x, y, z$, and $j_{\omega i}=\operatorname{Re}\left\{\Psi^{\dagger} \hat{\vec{\omega}} \times \hat{\vec{\sigma}} \Psi\right\}_{i}$ is the spin torque. According to the analogous ac charge current [20], we will start from the original Hamiltonian plus the induced field $\vec{H}_{1}$ and then calculate the spin current using the conventional definition. Now the spin current and spin torque both depend on $\vec{H}_{1}$. The conservation of spin current requires that the spin torque is zero or $\vec{j}_{w}\left(\vec{H}_{1}\right)=0$. Once $\vec{H}_{1}$ is determined, the conserved persistent spin current is obtained. We have used this approach to the hybrid ring and find the nonzero conserved persistent spin current.

From our results, several observations are in order: (i) Because the spin current is continuous, the spin current is nonzero in the SO region (near the normal part at least). It also exists when the normal region goes to zero so that the whole ring has the SOI. (ii) Besides the ring, the device can also be of other shapes, e.g., a disk device, and so on. So it is a generic feature that a pure persistent spin current appears in the SO coupling semiconductor as long as the size of the device is within the phase coherence length. (iii) There are a number of experiments that have been carried out to confirm the existence of a spin current either directly or indirectly [21]. There is also a proposal that a spin current may cause a spin torque that can be measured experimentally [22]. Since the persistent spin current is an equilibrium property, the above mentioned methods are not suitable. We note that the persistent charge current can be detected by measuring the magnetic field generated by the persistent current. It has been shown that the persistent spin current can also generate an electric field $[9,23]$. So this offers a way to detect the persistent spin current by detecting its induced electric field.

In summary, we show that a persistent spin current without an accompanying charge current exists in a semiconducting ring with only spin-orbit interaction so that the time-reversal symmetry is maintained. It is the spin Berry phase due to the SO interaction that induces the pure persistent spin current in the ring. We demonstrate the existence of the persistent spin current in a normal region of a SO coupling or normal hybrid device. It is an analog of the persistent charge current in the mesoscopic ring threaded by a magnetic flux. This persistent spin current describes the real spin motion and can be measured experimentally.

We gratefully acknowledge financial support from a RGC grant from the Government of HKSAR Grant No. HKU 7044/05P (J. W.), NSF-China under Grants No. 10474125 and No. 10525418 (Q.-f.S.), U.S. DOE under Grant No. DE-FG02-04ER46124, and NSF under No. CCF-052473 (X.C. X.).

*Electronic address: jianwang@hkusua.hku.hk

[1] I. Zutic, J. Fabian, and S. Das Sarma, Rev. Mod. Phys. 76, 323 (2004).

[2] S. Datta and B. Das, Appl. Phys. Lett. 56, 665 (1990).

[3] S. Murakami, N. Nagaosa, and S.-C. Zhang, Science 301, 1348 (2003).

[4] J. Sinova et al., Phys. Rev. Lett. 92, 126603 (2004).

[5] M. Büttiker, Y. Imry, and R. Landauer, Phys. Lett. 96A, 365 (1983); H.-F. Cheung et al., Phys. Rev. B 37, 6050 (1988).

[6] L.P. Levy et al., Phys. Rev. Lett. 64, 2074 (1990); V. Chandrasekhar et al., ibid. 67, 3578 (1991); D. Mailly et al., ibid. 70, 2020 (1993).

[7] D. Loss, P. Goldbart, and A. V. Balatsky, Phys. Rev. Lett. 65, 1655 (1990).

[8] J. Splettstoesser et al., Phys. Rev. B 68, 165341 (2003).

[9] F. Schütz et al., Phys. Rev. Lett. 91, 017205 (2003); Phys. Rev. B 69, 035313 (2004); P. Bruno and V. K. Dugaev, Phys. Rev. B 72, 241302(R) (2005).

[10] G. Usaj and C.A. Balseiro, Europhys. Lett. 72, 631 (2005).

[11] Recently, Rashba [Phys. Rev. B 68, 241315(R) (2003)] has found a nonzero spin current $I_{s}$ in an infinite twodimensional system with Rashba SOI in the thermodynamic equilibrium. In his opinion, this $I_{s}$ is not associated with real spin transport and, therefore, should be eliminated in calculating the transport current by modifying the definition of the spin current. This reference does not address the persistent spin current, the issue of this work.

[12] M. V. Berry, Proc. R. Soc. A 392, 45 (1984).

[13] A. G. Aronov and Y. B. Lyanda-Geller, Phys. Rev. Lett. 70, 343 (1993); Y. Lyanda-Geller, ibid. 71, 657 (1993).

[14] Q.-F. Sun and X.C. Xie, Phys. Rev. B 72, 245305 (2005).

[15] Y. Wang et al., Phys. Rev. Lett. 96, 066601 (2006).

[16] J. Shi et al., Phys. Rev. Lett. 96, 076604 (2006).

[17] J. Wang et al., Phys. Rev. B 74, 155307 (2006).

[18] F. E. Meijer et al., Phys. Rev. B 66, 033107 (2002).

[19] Note that the eigenlevel of $n=0$ is twofold degeneracy, and this level does not split at the nonzero $\alpha_{R}$ case.

[20] M. Büttiker, J. Phys. Condens. Matter 5, 9361 (1993).

[21] Y. K. Kato et al., Science 306, 1910 (2004); J. Wunderlich et al., Phys. Rev. Lett. 94, 047204 (2005); S. O. Valenzuela and M. Tinkham, Nature (London) 442, 176 (2006).

[22] T-W. Chen et al., Phys. Rev. B 73, 235309 (2006).

[23] Q.-F. Sun et al., Phys. Rev. B 69, 054409 (2004). 